\title{
Least Square
}

National Cancer Institute

\section{Source}

National Cancer Institute. Least Square. NCI Thesaurus. Code C94919.

A popular criterion used for a cost function, namely minimizing the sum of the squares of the differences between the data points and the analytical function (often a straight line) desired as a best fit. 